Man and Nature

L'homme et la nature

\title{
Vers une grammatologie du roman du XVIII ${ }^{\mathrm{e}}$ siècle
}

\section{Alexandre L. Amprimoz}

Volume 5, 1986

URI : https://id.erudit.org/iderudit/1011848ar

DOI : https://doi.org/10.7202/1011848ar

Aller au sommaire du numéro

Éditeur(s)

Canadian Society for Eighteenth-Century Studies / Société canadienne d'étude du dix-huitième siècle

ISSN

0824-3298 (imprimé)

1927-8810 (numérique)

Découvrir la revue

Citer cet article

Amprimoz, A. L. (1986). Vers une grammatologie du roman du XVIII ${ }^{\mathrm{e}}$ siècle. Man and Nature / L'homme et la nature, 5, 1-14.

https://doi.org/10.7202/1011848ar

Copyright (C Canadian Society for Eighteenth-Century Studies / Sociéte canadienne d'étude du dix-huitième siècle, 1986
Ce document est protégé par la loi sur le droit d'auteur. L'utilisation des services d'Érudit (y compris la reproduction) est assujettie à sa politique d'utilisation que vous pouvez consulter en ligne.

https://apropos.erudit.org/fr/usagers/politique-dutilisation/ 


\section{Vers une grammatologie du roman du XVIII e siècle}

\subsection{Introduction}

Ce que j'entends par encadrement c'est l'enveloppe d'une ouvre romanesque. Introductions, préfaces, avis de l'auteur et avis de l'éditeur sont des formes explicites de ce phénomène. $A$ ce niveau déjà, une certaine classification s'impose car l'encadrement peut aller du simple couvercle (La Nouvelle Marianne) à la forteresse (Paul et Virginie). Au-delà de la classification il faudra étudier la signification de certaines structures. L'avis de l'auteur dans le cas de Manon Lescaut est d'ordre rhétorique et non explicatif. Ce mince encadrement n'est-il pas, d'ailleurs, disséminé dans le texte? C'est ce genre de question qui engendre la définition d'un encadrement implicite.

Le cas des Illustres Françoises offre un exemple d'une certaine dissémination du cadre. De nouveau, plus qu'une architecture romanesque, c'est une certaine rhétorique de la lecture qu'il faut interroger et peut-être même mettre entre parenthèses.

Au-delà de la signification de l'encadrement il faudrait également définir et distinguer le roman du XVIII siècle. Ainsi La Princesse de Clèves a son minuscule couvercle et Adolphe, sa forteresse. Mais justement, entre ces deux bornes, ne se trouverait-il pas une évolution de l'encadrement? Et le roman au siècle des lumières, ne serait-il pas alors, plus qu'un récit, la justification de ce dernier? Il va sans dire que les résultats présentés constituent l'image d'une recherche 'en mouvement.' 


\subsection{Trois perspectives insatisfaisantes}

Trois méthodes de classement ont été utilisées jusqu'à aujourd'hui par les historiens de la littérature. La première tente d'établir des rapports entre le temps et les genres romanesques. Les travaux de Henri Coulet nous offrent un exemple de cette méthode. ${ }^{1}$ L'auteur de Le Roman jusqu'à la révolution voit trois époques dans l'évolution des oeuvres narratives du XVIII siècle. Il reconnaît lui-même l'arbitraire de ce genre de division. En effet, il écrit au sujet de sa 'troisième époque':

La date de 1760 n'a évidemment rien d'absolu, pas plus que les critères selon lesquels sont répartis les auteurs: Voltaire et Mme Riccoboni, rangés dans la période antérieure, ont écrit la plus grande partie de leurs romans après 1760; Baculard d'Arnaud, créateur du genre sombre, a commencé son œuvre dès 1745; Rousseau ouvre cette période finale tout aussi bien qu'il couronne la période précédente; enfin les catégories du genre romanesque qui existaient auparavant sont toujours pratiquées, l'exemple de Gil Blas, de La Vie de Marianne et surtout de Cleveland est loin d'avoir épuisé sa vertu. Mais aux environs de 1760, le roman traverse une crise grave et complexe. ${ }^{2}$

Malgré la formulation de ces réserves, Henri Coulet traite les genres comme s'ils avaient été pratiquement privilégiés par l'une ou l'autre des périodes considérées. De plus, il situe certains auteurs comme étant en dehors des genres qu'il établit, et ces auteurs sont les plus importants. Cette méthode est sans doute utile pour la documentation et pour l'établissement de manuels scolaires, mais elle présente des inconvénients quelque peu inquiétants qu'un résumé du classement de Henri Coulet semble facilement révéler: ${ }^{3}$

\begin{tabular}{|c|c|c|}
\hline $\begin{array}{l}\text { Epoque I } \\
\text { (1690-1715) }\end{array}$ & $\begin{array}{l}\text { Epoque II } \\
\text { (1715-1760) }\end{array}$ & $\begin{array}{l}\text { Epoque III } \\
\text { (1760-Révolution) }\end{array}$ \\
\hline $\begin{array}{l}\text { historique } \\
\text { galant } \\
\text { merveilleux } \\
\text { réaliste } \\
\text { philosophique }\end{array}$ & $\begin{array}{l}\text { Lesage } \\
\text { Marivaux } \\
\text { Prévost } \\
\text { Crébillon } \\
\text { de mours } \\
\text { sentimental } \\
\text { libertin } \\
\text { Montesquieu } \\
\text { Voltaire } \\
\text { La Nouvelle Hélö̈se }\end{array}$ & $\begin{array}{l}\text { réaliste } \\
\text { sentimental } \\
\text { libertin } \\
\text { philosophique } \\
\text { fantastique } \\
\text { du mal } \\
\text { Laclos } \\
\text { Sade } \\
\text { Restif de la Bretonne } \\
\text { Diderot }\end{array}$ \\
\hline
\end{tabular}


Aux inconvénients reconnus par l'auteur, il faut ajouter un certain nombre de difficultés d'ordre terminologique. Prenons l'exemple de Lesage. L'influence qu'il a subie du roman picaresque espagnol en fait déjà un cas délicat. ${ }^{4}$ De plus, Le Diable boiteux constitue un véritable problème car, publié en 1707 , il devrait être étudié dans la première période. L'existence de ces périodes empêche-t-elle que les ouvres d'un écrivain puissent se répartir sur plus d'une 'Epoque'? Mais on dira que l'édition qui fait autorité c'est la troisième (1736). Cela est vrai, mais parle-t-on du même livre? De plus, si le chef-d'œuvre Gil Blas est publié à partir de 1715 on comprend alors le sens de la révision profonde de la troisième édition de Le Diable boiteux.

D'autre part, en faisant un cas à part de Lesage, on finit par croire que le roman picaresque est sa spécialité. Mais il ne faut pas prendre le prétexte pour le texte. En effet, presque tous les romans de Lesage ont un cadre espagnol. On note au-delà du bien connu Gil Blas; Le Diable boiteux; L'Histoire du Guzman d'Alfarache (1732); L'Histoire d'Estevanille Gonzalès, surnommé le garçon de bonne humeur (1734); Le Bachelier de Salamanque, ou mémoires et aventures de Don Cherubin de la Ronda (1736).

Cependant le cadre ne doit pas tromper. Lesage est, avant tout, un romancier qui traite de mours. Dans son ouvrage Le Dilemme du roman au XVIII e siècle (Paris: P.U.F., 1963), Georges May nous rappelle ce point:

L'influence de La Bruyère semble être évidente, au moins autant que sur Le Diable boiteux (1707), dont c'est devenu une véritable platitude de la critique de dire qu'il est comme une seconde version, romancée et intégrée dans une trame romanesque élémentaire. (114-15)

Le rapport avec La Bruyère nous permet de souligner un postulat de l'évidence: tout roman de mours est un roman réaliste. Dans une classification des genres, le terme réaliste (qui se trouve dans les trois époques de Coulet) perd sa force, devient ambigu. D'ailleurs, pour ne pas créer de confusion avec les auteurs du XIXe siècle, il vaudrait mieux se servir du terme 'pré-réaliste.'

Utile pour la classification scolaire, l'explication des genres par la chronologie polarise la réalité du phénomène romanesque vers l'un de ses aspects - pas toujours le principal. L'examen du tableau qui résume le classement de Coulet révélerait d'ailleurs bien d'autres problèmes.

Une deuxième méthode de classement suit plus ou moins l'ordre chronologique qui, selon le mot d'Adamov, semble être le seul ordre logique possible.' Cette méthode peut être illustrée à partir de l'exemple 
de Jacques Vier. ${ }^{5}$ Sans suivre pas à pas l'évolution temporelle, Jacques Vier en tient compte juste assez pour établir un plan clair aux titres significatifs. L'idée de base est que l'on peut tracer l'évolution à partir des textes marquants. Voici le plan:

\section{Les Transformations du roman}

Première Partie: Le Prisme de l'aventure

Chapitre I - Robert Chasles et Les Illustres Françaises

Chapitre II - Alain René Lesage (1668-1747)

Chapitre III - L'Abbé Prévost d'Exiles (1697-1763)

Chapitre IV - Marivaux (1688-1763) romancier

Deuxième Partie: L'Attrait du vice

Chapitre V - Le Monde et la mode

Chapitre VI - Le Désert de l'amour

Chapitre VII - Les Délices de la conversation

Chapitre VIII - Les Affaires sérieuses

Troisième Partie: Les Puits de l'abîme

Chapitre IX - Les Liaisons dangereuses

Chapitre X - Nicolas-Edme Rétif de la Bretonne (1734-1806)

Chapitre XI - Le Marquis de Sade (1740-1814)

Quatrième Partie: L'Attrait de la vertu

Chapitre XII - Le Roman d'aventures édifiantes

Chapitre XIII - L'Arc-en-ciel du pathétique

Chapitre XIV - Bernardin de Saint-Pierre (1734-1814)

Chapitre XV - Le Roman féminin

Cette méthode a l'avantage de montrer une vue générale de l'univers romanesque au XVIII e siècle. Au point de vue thématique elle souligne des zones orbitales, ne plaçant pas ainsi les grands écrivains en dehors des genres. L'absence au niveau des titres de grands noms, comme celui de Voltaire, pose tout de même des difficultés. De plus, au niveau des sous-titres, on reconnaît certaines confusions de catégories. Par exemple 'le style épistolaire' n'est pas unique comme genre: les règles qui s'en dégagent pour Les Liaisons dangereuses ne sont pas les mêmes que celles qui fondent le jeu de Le Nouvelle Héloïse. Ainsi l'équivalence établie par Jacques Vier entre Les Liaisons dangereuses et le style épistolaire fausse un peu la base d'une théorie des genres. ${ }^{6}$ D'autre part, cette deuxième méthode reste trop près de l'histoire. Un classement devrait, grâce à une 
théorie des genres, déboucher sur un système de règles - sur une grammatologie romanesque.

On se doit enfin de signaler l'existence de méthodes partielles qui traitent d'aspects particuliers, souvent problématiques. C'est le cas de l'étude de Georges May qui s'intéresse aux rapports du roman et de la critique. C'est aussi le cas de Roger Mathé qui étudie l'exotisme. ${ }^{7}$

Ces méthodes sont très utiles car la multiplicité des points de vue permet de vérifier certaines structures romanesques. Cependant cette perspective métonymique, cette troisième théorie, est bien loin de tout principe de classement.

\subsection{L'Encadrement}

On pourrait passer en revue un grand nombre de théories littéraires et conclure qu'aucune ne semble conduire le lecteur vers une grammatologie romanesque du siècle des lumières. Deux exemples suffiront à évoquer rapidement les difficultés que l'on rencontre dans ce domaine. Ainsi, selon Wayne C. Booth, 'true novels must be realistic. ${ }^{8}$ Nous voilà donc réduits, une fois de plus, à la première méthode (1.0). Il faut aussi noter le danger qui consiste à faire précéder l'analyse de l'herméneutique. Le même genre de piège est inscrit dans la méthode de Northrop Frye qui fonde tout classement possible sur le rapport infériorité/supériorité: lecteur/héros. ${ }^{9} \mathrm{Il}$ est clair que les composantes psychologiques et morales du roman du XVIII ${ }^{e}$ siècle n'entraîneraient que des discussions interminables dans le domaine du classement. Peut-on, par exemple, répondre facilement à la question suivante: Manon Lescaut est-elle inférieure ou supérieure au lecteur? C'est donc une approche plutôt formelle de l'histoire littéraire qui peut ouvrir la voie aux recherches dans ce domaine.

Le premier de ces aspects formels qui se présente à l'esprit est donc l'encadrement, c'est-à-dire l'enveloppe de l'ouvre romanesque. Comme je l'ai déjà signalé, l'analyse des romans montre que ce phénomène va du simple couvercle, parfois fin jusqu'à transparence, passe par le coffre moyen et peut atteindre les dimensions d'une forteresse. L'introduction de la notion d'encadrement est indispensable car bien qu'elle fasse partie du discours, elle ne peut être assimilée à ce dernier. Il faut insister: certaines de ces formes sont explicites: introductions, préfaces, avis de l'auteur, avis de l'éditeur, etc. D'autres sont implicites. Il s'agit maintenant, à l'aide d'exemples, de distinguer différents degrés d'encadrement. 


\subsection{Degré zéro d'encadrement}

C'est le couvercle le plus fin, le plus transparent. L'absence de préface ou d'avis montre que l'œuvre se présente pratiquement sans intentions avouées. C'est, par exemple, là le cas d'oeuvres de première classe telles que Candide et Le Neveu de Rameau. Ce dernier exemple peut être qualifié de sotie, implicite, parce que ce genre était encore censuré à l'époque. L'une des fonctions du degré zéro d'encadrement semble donc le détour de l'interdit. Dans le cas de Candide l'interdit du contenu paraît dominer, dans celui du Neveu de Rameau c'est d'abord l'interdit d'une forme que l'on veut contourner.

Passons à une fonction à première vue plus innocente du phénomène étudié. Quand on est en présence d'un texte qui est une imitation d'un chef d'œuvre connu, on a souvent à faire au degré zéro d'encadrement. C'est le cas de La Nouvelle Marianne. ${ }^{10}$ En effet, la préface ne semble qu'une précaution, une conséquence d'un fait historique: la proscription des romans. ${ }^{11}$ Depuis les années trente il fallait être prudent. Dans ce domaine l'abbé Lambert suit la règle:

A ces sentiments (dit la Baronne), je mêle les réflexions qui les ont d'ordinaire accompagnées. Le bon sens, la religion et l'honneur, me les inspiroient. (A3)

On peut donc écarter cette préface. Le titre est le véritable encadrement. Quant au roman, il s'ouvre de la manière la plus claire:

J'avois déjà atteint ma vingt-deuxième année, et je n'étois pas encore instruite de quel sang je tirois mon origine. (A4)

C'est, bien sûr, le parallèle avec l'œuvre de Marivaux qui commence.

On est aussi en présence du degré zéro quand le roman a la voix forte et solitaire. L'auteur écrit: il ne se berce pas dans les théories romanesques. C'est le cas du Marquis de Sade avec Les Infortunes de la vertu. Point de préface mais une mise en garde directe au début de l'ouvre:

Il vaut infiniment mieux prendre parti parmi les méchants qui prospèrent que parmi les vertueux qui périssent? Il est donc important de prévenir ces sophismes de la philosophie. ${ }^{12}$

Il s'agit donc d'une préoccupation morale, mais dans la foulée car le livre se ferme sur: 'Fini au bout de quinze jours le 8 juillet $1787 .^{\prime 13}$

En général, on peut conclure que le degré zéro d'encadrement s'applique aux œeuvres qui ont plus de force - c'est-à-dire sens qui se cherche - 
que de signification. Ces dernières ont le pas rapide quand elles puisent dans la mémoire une ligne déjà tracée. Mais lorsqu'elles suivent le cours de l'imagination, elles ne font que la prendre en filature et tendent alors vers une écriture automatique. Ce sont du moins là les illusions, car le degré zéro d'encadrement semble toujours avoir pour fonction le détour d'une censure explicite ou même subtile.

\subsection{Le Premier Degré d'encadrement}

Nombreuses sont les œuvres qui entrent dans cette catégorie. Pour montrer la teneur de ce niveau nous utiliserons les romans de Crébillon fils. La raison de ce choix peut facilement se justifier: chaque roman a une forme différente des autres, tout en conservant le même degré $\mathrm{d}^{\prime}$ encadrement. Ceci indique déjà qu'il n'y a pas obligatoirement isomorphisme entre genre et encadrement. Le premier degré est donc celui de la sobre préface parfois précédée par une dédicace. Si je place dans cette catégorie l'ouvre de Crébillon, c'est que, malgré l'ironie de l'auteur au sein des textes, ceux-ci constituent encore des unités. Au point de vue encadrement, l'ouvre romanesque de Crébillon est totalement différente de celle de Diderot. Rappelons que nous analysons surtout les formes et que cette étude tente d'éviter les interprétations de la dialectique qui s'établit entre l'auteur et le lecteur.

Pour revenir à ce premier degré il suffit de feuilleter les Oeuvres complètes de Crébillon (Londres, 1779, 7 volumes). On voit, par exemple, un roman sous forme de mémoires: Les Egarements du coeur et de l'esprit précédé d'une lettre dédicace (à son père) et d'une préface assez courte. D'autre part, on voit Lettres de la marquise de $M^{* * *}$ au comte de $R^{* * *}$ précédé d'un extrait d'une lettre de madame de ${ }^{* \star *}$ à $\mathrm{M}$. de ${ }^{* * *}$ qui sert de préface déguisée. Il semble donc que Crébillon ne peut éviter ce premier degré d'encadrement, ce désir de détour. En effet, même dans La Nuit et le moment ou Le Hasard au coin du feu, qui sont des romans sous forme de dialogues, on ne trouve pas la nature immédiate du degré zéro de l'encadrement.

Indépendamment du genre de la forme, le premier degré d'encadrement est caractérisé par une préface explicite ou implicite. Il y a, d'ailleurs, une coupure entre la préface et le corps de l'oeuvre, ce qui permet de ne pas confondre ce niveau avec le degré zéro.

Le premier degré d'encadrement indique une inquiétude, une réflexion; plus une intention qui donne tous les signes d'un mode d'emploi. La préface fait du roman un manuel qui, d'une manière directe ou indirecte, 
impose une méthode de lecture. En ce sens on s'éloigne d'un récit primitif, donc du genre romanesque, pris dans la signification de son origine.

\subsection{Les Epaisseurs successives}

La première de ces épaisseurs est celle qui recouvre des oeuvres comme Manon Lescaut de l'abbé Prévost. Il s'agit donc d'une superposition des degrés définis dans 2.1 et 2.2. En effet, on trouve tout d'abord 'Avis de l'auteur des Mémoires d'un Homme de Qualité'14 qui, étant l'équivalent d'une préface, définit un premier degré d'encadrement. Ensuite, on est en présence d'une note de l'éditeur, mais l'ultériorité de cette dernière nous apprend 'qu'on s'est déterminé à le purger d'un grand nombre de fautes grossières' et que 'on y a fait aussi quelques additions qui ont paru nécessaires pour la plénitude d'un des principaux caractères. ${ }^{\prime 15}$ Tout ceci montre une évolution du texte qui contribue à l'épaisseur de l'encadrement. Le cas de Prévost n'est, bien sûr, pas une exception: que l'on songe à nouveau aux remaniements de Le Diable boiteux, aux différences profondes qui marquent les deux textes de Lesage (1 ${ }^{\text {ère }}$ et $3^{\text {ème }}$ édition). Ensuite l'on trouve dans le texte de Manon Lescaut quelques pages introductives que l'on classe dans le degré zéro d'encadrement. ${ }^{16}$ Ces pages n'indiquent point une intention comme 'L'Avis de l'auteur' mais placent le récit dans son contexte.

Ce premier niveau d'épaisseur conduit la lecture vers des structures plus complexes d'encadrement. Avant d'en commencer l'analyse, il faut signaler que l'on atteint ici une conception tout à fait différente du livre. Il devient de plus en plus difficile de distinguer la force de la signification. Le jeu devient projet, calcul, composition qui indique l'attraction de l'œuvre pour celui qui l'étudie. En d'autres mots, l'intention subjective de l'écriture est le phénomène que doit analyser le lecteur. Il s'agit là d'un problème assez grave: la lecture de Manon Lescaut peut-elle être immaculée d'interprétation? L'analyse de la forme nous montre une épaisseur d'intention face à un récit. La question de compatibilité entre ces deux parties est une étude d'unité de forme; mais la réponse à cette question implique une interprétation. En effet, comment peut-on résoudre le problème posé par Prévost lui-même:

Un lecteur sévère s'offensera peut-être de me voir reprendre la plume, à mon âge, pour écrire des aventures de fortune et d'amour; mais si la réflexion que je viens de faire est solide, elle me justifie; si elle est fausse, mon erreur sera mon excuse. (6-7) 
On comprend, face au texte de Manon Lescaut, pourquoi la critique non-formelle peut être réduite à un conflit d'interprétations. Il s'agit simplement d'être conscient du dilemme.

Il est clair que le projet en question n'est pas simple. Chacun des critères exposés offre plutôt une méthode qu'une classification. L'épaisseur, par exemple, servirait dans un recensement assez utile. On aurait, en suivant ce principe, trois classes. Les deux premières ('zéro' comme La Nouvelle Marianne et 'un' comme les oeuvres de Crébillon fils) sont faciles à reconnaître. Mais la troisième irait du minimum de Manon Lescaut au maximum de Paul et Virginie de Bernardin de Saint-Pierre. ${ }^{17}$ On vérifiera facilement ce point si l'on établit le rapport entre encadrement et texte proprement dit:

Préambule pp. $27-80$

54 pages

Avant-propos

(Editions de $1788-89$ et 1800 )

2 pages

Avis sur cette édition (1789)

10 pages

d'où:

Encadrement

Paul et Virginie (pp. 81-175)

66 pages

95 pages

Jusqu'à présent deux approximations ont été admises: on n'a guère tenu compte de la périodisation et de la dissémination du cadre dans le tableau. En effet, il s'agit là encore d'un aspect introduisant une coupure de l'unité. Une première loi peut maintenant être dégagée (bien sûr les exceptions ne manquent pas, mais la grammatologie romanesque n'est pas une géométrie): L'unité de l'oeuvre dépend de l'épaisseur de son encadrement. L'exemple de Paul et Virginie illustre cette proposition.

\subsection{Dissémination du cadre}

Les notions d'épaisseur, d'encadrement et d'unité formelle de l'oeuvre conduisent automatiquement à la notion de dissémination. On peut définir la dissémination du cadre comme l'ensemble des ramifications de l'épaisseur (c'est-à-dire de l'intention avouée) dans le texte. Il serait difficile et peut-être insensé de prouver qu'il existe un seul roman qui ne soit pas soumis à ce phénomène; mais ici aussi c'est la notion de degré qu'il faut considérer. Il y a des disséminations plus apparentes que d'autres et une ouvre peut en contenir plusieurs. Encore une fois, on tentera 
d'établir des classes, allant des formes les plus simples aux structures les plus complexes.

\subsection{La Périodisation}

La division d'un roman en parties peut bien être un choix de composition. Mais la décision qui fait que ces parties soient publiées successivement à intervalles plus ou moins réguliers signale déjà une coupure dans la notion de périodisation. Ainsi on pourrait classer un certain nombre de romans allant de La Vie de Marianne à La Nouvelle Héloüse. Une distinction entre les œuvres publiées en parties et les œuvres dont toutes les parties sont publiées ensemble est donc possible et peut s'avérer même fertile.

\subsection{La Dissémination et le pluriel du récit}

Nous avons introduit la notion d'encadrement comme un sous-ensemble du discours. De plus, une des caractéristiques du roman au XVIIIe siècle semble être la pluralité des récits secondaires qui composent les textes. La suite naturelle de notre analyse nous porte donc vers une dialectique qui pousse le problème grammatologique un pas plus loin.

3.0 La Grammatologie romanesque comme étude des partitions discursives et narratives

Dans une première distinction les termes peuvent être redéfinis par rapport à l'épaisseur de l'intention: 1) Encadrement: Partie du discours établissant les intentions avouées du narrateur-auteur et ses réactions à la force des récits. 2) Histoire: d'abord le pluriel des récits. Il faut ensuite considérer les intentions et les réactions des personnages en incluant les narrateurs non-auteurs.'

\subsection{Premier Système de dissémination: le réseau de fables}

On définira cette catégorie à l'aide du roman de Robert Challes: Les Illustres Françoises (ou Robert Chasles, Les Illustres Françaises). ${ }^{18}$ L'architecture de cette oeuvre est du même genre que celles qui ont été consacrées par les recueils de fables célèbres (Décaméron, Mille et une nuits, 
etc). Le volume semble être basé sur le chiffre sept comme L'Heptaméron. Voici la répartition Encadrement/Histoire:

\section{Encadrement: Préface}

Des Frans rencontre son ami Des Ronais à Paris.

Des Frans et Des Ronais ne se sont pas vus pendant sept ans.

Histoire:

Fable I. Narrateur: Des Ronais

Monsieur Des Ronais et Mademoiselle Dupuis

Fable II. Narrateur: Des Ronais

Monsieur de Contamire et Angéline

Fable III. Narrateur: Terny

Monsieur de Terny et Mademoiselle de Bernay

Encadrement: Terny nous apprend que la lettre lue par Des Ronais était effectivement celle qu'il avait adressée à Mlle de Vernay.

Histoire:

Fable IV. Narrateur: Des Frans

Monsieur de Jussy et Mlle Fenoüil

Fable V. Narrateur: Dupuis

Monsieur de Prex et Mlle de L'Epine

Fable VI. Narrateur: Des Frans

Monsieur des Frans et Silvie

Fable VII. Narrateur: Dupuis

Monsieur Dupuis et Madame de Londé

Encadrement: Les mariages
Des Frans
Mme de Mongey
Dupuis
Mme de Londé
Des Ronais
Mlle Dupuis

Une analyse plus poussée montrerait une architecture savante s'établissant entre les histoires et les commentaires des personnages. Ceci n'est pas le but de cette étude; on se contentera de noter simplement le rôle que joue au sein des structures narratives le discours. Ce dernier met en valeur la notion d'encadrement. On peut donc s'aventurer vers une nouvelle règle: Quand l'encadrement établit une répartition claire dans l'histoire c'est le reste du discours qui offre et développe une dialectique 
complexe du récit. Il y aurait donc une catégorie de romans (système de fables) dont la clarté de l'encadrement impliquerait une dissémination aux structures précises. Notons aussi que dans notre exemple, les possibilités combinatoires ne sont pas épuisées.

\subsection{Deuxième Système de dissémination: l'épopée du prédictif}

La notion de 'prédictif' a été mise au point par Tzvetan Todorov. ${ }^{19}$ Un exemple précis montrera le mode prédictif au niveau différentiel. En effet, le prédictif est beaucoup plus une promesse d'un récit que tout autre chose. C'est souvent le cas de La Vie de Marianne. Ainsi Marivaux termine souvent une partie avec une promesse:

Je vous dirai le reste dans la septième partie, qui, à deux pages près, débutera, je le promets, par l'histoire de la religieuse, que je ne croyais pas encore si loin quand j'ai commencé cette sixième partie-ci. ${ }^{20}$

Cet exemple ne donne pas une vue totale de la richesse du prédictif. En effet, il faut l'approfondir à partir de La Grammaire du Décaméron où elle revêt un aspect assez simple qui reflète la psychologie élémentaire de l'oeuvre. Comment pourrait-on classer les romans par rapport à la notion de prédictif?

\subsection{Prédictif et pluriel de l'encadrement}

Encore une fois, c'est la forme qui offre les indications les plus précieuses. Ce genre est constitué par des romans divisés en chapitres. De plus, chaque chapitre a un sous-titre qui, en une ou deux phrases, le résume. Dans Gil Blas cette méthode est clairement employée. Dans Candide le prédictif est plus ambigu; dans Les Bijoux indiscrets il est simplement déroutant. Le prédictif, qu'il soit à l'état différentiel ou intégral, va d'un emploi neutre vers un emploi ironique. Il serait une notion utile pour établir une rhétorique de l'ironie dans les romans considérés.

\subsection{Troisième Système de dissémination: le prédictif mine du cadre}

Il est évident que jusqu'à présent on a considéré le prédictif comme étant le privilège de l'auteur-narrateur. Pourtant ce mode produit de véritables explosions dans Jacques Le Fataliste. C'est qu'il est passé de l'auteur- 
narrateur aux personnages-narrateurs et même aux personnages-nonnarrateurs.

Cette remarque et ce qui précède nous conduisent à la loi suivante: $L a$ pluralité du prédictif tend vers un maximum qui produit un effet d'indétermination totale.

\subsection{Conclusion}

Une grammatologie romanesque aboutirait à un manuel exposant le nombre limité de règles qui régissent la composition des romans considérés. Cependant ce recensement devrait se faire également à l'état différentiel. Tout d'abord, au niveau du récit, où il montrerait les écarts entre logique prédictive et logique narrative; puis, par rapport à certaines données fondamentales de l'œuvre, comme le temps et l'espace.

\section{ALEXANDRE L. AMPRIMOZ Brock University}

\section{Notes}

1 Henri Coulet, Le Roman jusqu'à la révolution, Tome I: Histoire du roman en France, Seconde édition revue (Paris: Colin 'Collection U,' 1967)

2 Ibid., 419

3 Ibid., 286-518

4 Cette étude a été approfondie par G. Reynier dans son livre Le Roman réaliste au $\chi V I I I^{e}$ siècle. On se rend compte, en particulier, de la lenteur de l'influence espagnole.

5 Jacques Vier, Histoire de la littérature française, XVIII siècle, Tome II: Les Genres littéraires et l'éventail des sciences humaines (Paris: Colin 1970), 334-580

6 Ibid., 1046-8

7 Roger Mathé, L'Exotisme: d'Homère à Le Clézio (Paris: Bordas 1972), 93-115

8 Wayne C. Booth, The Rhetoric of Fiction (Chicago: University of Chicago Press 1961)

9 Northrop Frye, Anatomy of Criticism: Four Essays (Princeton, NJ: Princeton University Press 1957), 3-70 
10 Roman publié à La Haye, Chez Pierre de Hondt (1751): La Nouvelle Marianne, ou les mémoires de la Baronne de ${ }^{\star \star \star}$, écrits par elle-même. Première partie. L'auteur véritable en est l'abbé Claude François Lambert.

11 A ce sujet, voir l'ouvrage de Georges May (cité plus haut dans le texte). En particulier le Chapitre III: 'La proscription des romans,' 75-105.

12 Marquis de Sade, Les Infortunes de la vertu, introduction par Gilbert Lely (Paris: 10/18, 1968), 22

13 Ibid., 187

14 Edition Garnier de F. Deloffre et R. Picard (Paris, 1965), 3-7

15 Ibid., 8

16 ibid., 9-17

17 Bernardin de Saint-Pierre, Paul et Virginie, Edition de Robert Mauzi (Paris: Garnier-Flammarion, 1966)

18 L'orthographe de ces noms est discutée dans l'étude de Frédéric Deloffre: $\mathrm{La}$ Nouvelle en France à l'âge classique (Paris: Didier, 1967).

19 En particulier 'La Grammaire du récit' in Poétique de la prose (Paris: Seuil, 1971): Grammaire du Décaméron (Paris et La Haye: Mouton 1969).

20 Marivaux, La Vie de Marianne, Edition de F. Deloffre (Paris: Garnier 1963), 318 